\title{
Registered Dietitian Wellness Insurance Benefit Makes a Difference in Adult Weight Management: A Pre-Post Study
}

\author{
Linda Snetselaar ${ }^{1}$, Karen L. Smith ${ }^{1}$, Donna Hollinger ${ }^{1}$, Esther Myers ${ }^{2}$, Gwen Murphy ${ }^{3}$, \\ Laura Goettinger Qualls ${ }^{4}$
}

${ }^{1}$ Department of Epidemiology, University of Iowa, Iowa City, USA; ${ }^{2}$ American Dietetic Association, Chicago, USA; ${ }^{3}$ Department of Community and Family Medicine, Duke University Medical Center, Durham, USA; ${ }^{4}$ Blue Cross and Blue Shield of North Carolina, Durham, USA.

Email: linda-snetselaar@uiowa.edu

Received September $1^{\text {st }}$, 2011; revised October $15^{\text {th }}$, 2011; accepted October $22^{\text {nd }}, 2011$.

\begin{abstract}
Registered Dietitian $(R D)$ services as part of insurance wellness programs offer a promising venue for improving public health. This study was conducted to evaluate the effectiveness of RD nutrition counseling services provided as part of an insurance benefit on body weight and associated health parameters. Eligible members could enroll to receive 6 RDs visits a year for assistance with weight management. The study RDs were randomized into either Usual Care (UC) or Lifestyle Case Management (LCM) groups. Body weight, waist circumference, and systolic and diastolic blood pressure measurements of program enrollees were evaluated for between group as well as start and end program comparisons. There was a statistically significant difference in the number of RD follow-up visits between the two groups as LCM patients had more RD contact than UC patients. Weight and waist circumference changes from baseline to end of study show statistically significant changes with a trend for improvement in systolic blood pressure. Additionally, a clinically significant reduction in weight was achieved in a quarter of program enrollees. In conclusion this study shows that through a coordinated health promotion program RDs' services are of value to an insured population.
\end{abstract}

Keywords: RD Nutrition Counseling, Insurance Benefit, Weight Loss, Blood Pressure

\section{Introduction}

Accumulating evidence supports the benefit of Medical Nutrition Therapy (MNT) provided by RDs in improving health related parameters. Whether it is one counseling session or multiple counseling sessions, RD services improve chronic disease management [1-5]. Climbing obesity rates [6] contribute to rises in health care costs [7]. Since a charge of wellness programs is to prevent chronic diseases such as heart disease and diabetes, insurance companies are exploring the effectiveness of wellness benefits. A weight loss of $5 \%-10 \%$ can often delay the onset or reduce the severity of these diseases $[8,9]$. Provision of RD nutrition counseling services as part of insurance program wellness benefits presents an opportunity to reduce body weight and therefore improve the health of Americans. The goal of this project was to compare the effectiveness of $\mathrm{RD}$ counseling services offered as an insurance benefit, before and at the end of program completion on weight, waist circumference, systolic and diastolic blood pressure. A secondary goal was to assess if motivational interviewing (MI) has additional benefit when added to behavioral counseling strategies.

\section{Methods}

\subsection{Study Design}

This is a pre-post study involving RDs who are members of the American Dietetic Association Dietetics Practice Based Research Network (DPBRN). Through a wellness program sponsored by Blue Cross and Blue Shield of North Carolina (BCBSNC), study RDs were randomized within geographic region to two study groups. The two groups were UC or LCM. For RDs who worked in a joint practice, the entire practice was randomized to the same 
study assignment. RDs in both groups received training in study protocol and behavioral counseling skills. In addition, RDs in the LCM group received training in MI. Once randomized, RDs in each arm of the study recruited patients. Both arms of the study included up to 6 reimbursable visits per year.

\subsection{Study Eligibility}

Select BCBSNC enrollees were eligible for membership in Member Health Partnerships (MHP), a health plan benefits wellness program. Eligible study participants were MHP program participants age 18 or over with a body mass index (BMI) of 24.5 or higher who had at least one face-to-face encounter with a study RD between March 2006 and November 2008 and consented to participate in the study. Individuals who were pregnant, had outpatient renal dialysis or bariatric surgery, or were treated for cancer were not eligible to participate in the study. Study participants who experienced these events at any time during the study were terminated from the study at the time of the event. The study ran from March 2006 until July 2009.

\subsection{Recruitment}

All participants in the MHP program were required to complete a program enrollment survey. This survey was used by the health plan to send an individualized report with lifestyle change suggestions to each member. Upon enrollment in MHP, the members became eligible for $6 \mathrm{RD}$ visits per year and 4 physician visits per year to assist with weight loss. During the first two years of the study, the health plan sent two letters to members in the catchment area where each study RD (UC and LCM) practiced to encourage members to make an appointment with the RD. For each patient seen, the $\mathrm{RD}$ would then verify member eligibility for both the $\mathrm{RD}$ benefit and for the study. The study RD obtained informed consent from the enrollee.

\subsection{Study Population}

A total of 574 enrollees consented to participate in the study. Of these 559 were eligible, while 15 (2.6\%) were excluded for the following reasons: 4 did not have a faceto-face RD encounter with a documented height and weight; 7 had baseline BMI levels less than 24.5, and 4 were not participating in the MHP program. While the original intent was to follow patients for 2 full years, it became clear that the number of patients with 2 years of data would be a small number. Therefore, the final study population represented here was comprised of the 239 study participants who participated in the study for at least one year. This population represented slightly less than half (42.8\%) of the eligible 559 participants.

\subsection{Study Intervention}

All study patients in both LCM and UC received the standard MHP program intervention as well as MNT. In addition, patients in LCM received a MI counseling approach. The standard MHP program intervention included access to telephonic nursing support, educational materials, behavioral change tools as well as discounts on blood pressure and heart rate monitors. Medical Nutrition Therapy is part of the Nutrition Care Process (NCP) which includes the following steps: nutrition assessment, nutrition diagnosis, nutrition intervention, and nutrition monitoring and evaluation [10]. The RDs in this study monitored and evaluated outcomes using NCP.

\subsection{UC and LCM Training}

Both UC and LCM RDs were instructed on study procedures including recruitment, reimbursement, and outcomes documentation. MNT Assistant software was provided to each RD to use for study data collection. All RDs received training in current evidence-based weight management including topics such as genetic factors, metabolic considerations, and behavioral implications of obesity. They also received information on adult learning principles and goal setting. Both groups of RDs were trained on the evidence-based nutrition counseling theories and strategies outlined in the NCP. In addition, LCM RDs had separate training in MI with the goal of enhancing the way in which RDs communicated with their patients.

\subsection{Statistical Methods Analysis}

This study design is a pre-post analysis of a single treatment group. Study participants who had missing or invalid responses at either measurement point were excluded from the analyses. Thresholds for valid responses were established based on the empirical experience of the authors and registered dietitians. The valid range of values was 45.36 $226.80 \mathrm{~kg}$ (100 - $500 \mathrm{lbs)}$ for weight; 81.3 - $203.2 \mathrm{~cm}$ (32 80 inches) for waist circumference; $70-250 \mathrm{~mm} \mathrm{Hg}$ for systolic blood pressure; 40 - $120 \mathrm{~mm} \mathrm{Hg}$ for diastolic blood pressure; and an average weight loss of no greater than 0.68 $\mathrm{kg}(1.5 \mathrm{lbs})$ per week. Outcome measures are described in detail in a previous publication [11]. Data were analyzed using PC SAS version 9.1 (SAS Institute, Cary, NC). A result was considered to be statistically significant if the observed significance level ( $p$ value) was less than 0.05 . Differences were analyzed using t-tests.

\section{Results}

\subsection{Baseline Characteristics}

Descriptive statistics for relevant baseline characteristics 
of the study population are provided in Table 1. Age and gender were obtained from BCBSNC's membership records, and diabetes was self-reported by the study participant on the MHP program enrollment survey. All other data were obtained from the RDs.

\subsection{Study Duration and Frequency of Visits}

The first set of measurements was taken at the first RD counseling session following enrollment in the study, termed "program entry". The final set of measurements, termed, "program completion" was taken during the last $\mathrm{RD}$ counseling session completed by each patient. Because all study participants took part in this intervention as an insurance wellness benefit, the duration and number of RD visits varied from patient to patient. The average duration in the study was almost two years (mean = 21.3 months, SD $=5.3$ ) and was comparable among those in the Usual Care (mean $=21.1$ months, $\mathrm{SD}=5.4$ ) and LCM (mean $=21.5$ months, $\mathrm{SD}=5.3$ ) groups. The average number of visits during the study period was 7.8 ( $\mathrm{SD}=3.8$ ) and was significantly higher among the LCM group (mean $=9.2, \mathrm{SD}=4.2$ ) compared to the UC group (mean $=6.5, \mathrm{SD}=2.8), t(237)=5.8, p<0.001$.

\subsection{Missing/Invalid Data}

The percentage of study participants who had missing values was $0 \%$ for weight, $23.4 \%$ (56/239) for waist circumference, and $33.5 \%$ for blood pressure (80/239). The thresholds for valid data were described previously in the Analyses section. Of those who had documented values, weight was invalid $0.4 \%(1 / 239)$ of the time; waist circumference was invalid $8.2 \%$ (15/183) of the time; systolic blood pressure was invalid 2.5\% (4/159) of the time; and diastolic blood pressure was invalid 3.1\% (5/159) of

Table 1. (a) Baseline characteristics metric version; (b) baseline characteristics English measurements.

(a)

\begin{tabular}{lccc}
\hline & \multicolumn{2}{c}{ Baseline Characteristics } \\
\cline { 2 - 4 } & All & Usual Care & LCM \\
\hline Study participants & 239 & 119 & 120 \\
Age; mean & $46.5(\mathrm{~N}=239$; SD = 10.8) & $46.3(\mathrm{~N}=119 ; \mathrm{SD}=10.1)$ & $46.8(\mathrm{~N}=120 ; \mathrm{SD}=11.5)$ \\
Gender; percent female & $82.0 \%(196 / 239)$ & $81.5 \%(97 / 119)$ & $82.5 \%(99 / 120)$ \\
Weight in kilograms; mean & $95.1(\mathrm{~N}=239 ; 22.4)$ & $96.1(\mathrm{~N}=119 ; \mathrm{SD}=22.3)$ & $94.1(\mathrm{~N}=120 ; \mathrm{SD}=22.4)$ \\
BMI; mean & $34.4(\mathrm{~N}=239 ; \mathrm{SD}=7.1)$ & $34.4(\mathrm{~N}=119 ; \mathrm{SD}=7.0)$ & $34.3(\mathrm{~N}=120 ; \mathrm{SD}=7.1)$ \\
Waist circumference in cm among females; mean & $105.7(\mathrm{~N}=166 ; \mathrm{SD}=17.5)$ & $109.0(\mathrm{~N}=73 ; \mathrm{SD}=18.5)$ & $103.4(\mathrm{~N}=93 ; \mathrm{SD}=16.3)$ \\
Waist circumference in cm among males; mean & $117.6(\mathrm{~N}=39 ; \mathrm{SD}=16.3)$ & $117.1(\mathrm{~N}=18 ; \mathrm{SD}=17.0)$ & $118.1(\mathrm{~N}=21 ; \mathrm{SD}=16.0)$ \\
Diabetes & $13.7 \%(31 / 226)$ & $14.3 \%(16 / 112)$ & $13.2 \%(15 / 114)$ \\
\hline
\end{tabular}

(b)

\begin{tabular}{lccc}
\hline & \multicolumn{3}{c}{ Baseline Characteristics } \\
\cline { 2 - 4 } & All & Usual Care & $L C M$ \\
\hline Study participants & 239 & 119 & 120 \\
Age (years) & $46.5(10.8)(\mathrm{N}=239 ; \mathrm{SD}=10.8)$ & $46.3(10.1)(\mathrm{N}=119 ; \mathrm{SD}=10.1)$ & $46.8(11.5)(\mathrm{N}=120 ; \mathrm{SD}=11.5)$ \\
Gender (percent female) & $82.0 \%(196 / 239)$ & $81.5 \%(97 / 119)$ & $82.5 \%(99 / 120)$ \\
Weight (pounds) & $209.6(\mathrm{~N}=239 ; 49.4)$ & $211.8(\mathrm{~N}=119 ; \mathrm{SD}=49.3)$ & $207.5(\mathrm{~N}=120 ; \mathrm{SD}=49.5)$ \\
BMI (kg/m $\left.{ }^{2}\right)$ & $34.4(\mathrm{~N}=239 ; \mathrm{SD}=7.1)$ & $34.4(\mathrm{~N}=119 ; \mathrm{SD}=7.0)$ & $34.3(\mathrm{~N}=120 ; \mathrm{SD}=7.1)$ \\
Waist circumference (inches) among females & $41.6(\mathrm{~N}=166 ; \mathrm{SD}=6.9)$ & $42.9(\mathrm{~N}=73 ; \mathrm{SD}=7.3)$ & $40.7(\mathrm{~N}=93 ; \mathrm{SD}=6.4)$ \\
Waist circumference (inches) among males & $46.3(\mathrm{~N}=39 ; \mathrm{SD}=6.4)$ & $46.1(\mathrm{~N}=18 ; \mathrm{SD}=6.7)$ & $46.5(\mathrm{~N}=21 ; \mathrm{SD}=6.3)$ \\
Diabetes incidence (percent with disease) & $13.7 \%(31 / 226)$ & $14.3 \%(16 / 112)$ & $13.2 \%(15 / 114)$ \\
\hline
\end{tabular}


the time.

\subsection{Comparison of Pre-Post Change}

Comparison of Pre-Post Changes in both UC and LCM Groups Combined Table 2 indicates the percentage of participants who were able to achieve clinically significant improvements. Looking at the entire group of participants who participated in the MHP program and were enrolled in this study (Figure 1), the program achieved important pre-post differences. Weight and waist circumference changes from baseline to end of study show statistically significant changes with a trend for improvement in systolic blood pressure.

\section{Discussion}

The BCBSNC study data indicate that inclusion of a RD counseling insurance benefit made it possible to achieve clinically significant results in weight loss in a quarter of program enrollees. The ability to affect this proportion of people has major implications in terms of decreasing risk for chronic diseases such as diabetes, coronary heart disease and cancer. Additionally changes were seen in diastolic blood pressure with nearly half of the enrollees in this program having a clinically significant change in diastolic blood pressure. This important change has the potential to help in reduction of blood pressure medications resulting in cost savings. It also may be of value in reducing renal progression and reducing incidence of cardiovascular diseases. The duration and number of RD visits varied from patient to patient. This variability is inherent in practice-based studies. There was a statistically significant difference in the number of follow-up visits between the two groups. LCM patients had more RD contacts than UC patients. We hypothesize that the greater number of average follow-up visits for the LCM group can be attributed to an MI counseling approach. Since an increased number of counseling sessions has been associated with weight loss, improved fasting plasma glucose, total cholesterol and triglyceride levels [2], the higher rate of return visits achieved with a MI counseling approach is noteworthy in terms of chronic disease prevention and management. A limitation of the study included lack of screening of RDs for MI counseling training prior to randomization. Due to the exponential growth of interest in MI [12], it became apparent as the study progressed that some RDs randomized to the UC group were already familiar with MI or had sought out MI training over the course of the study. We suggest that this may have diluted outcome differences between the two groups. Another limitation of the study was the learning curve for becoming proficient with MNT assistant. We hypothesize that this time in-
Table 2. Percentage of study participants achieving clinically significant reductions.

\begin{tabular}{cccc}
\hline & All & Usual Care & LCM \\
\hline Weight & $24.8 \%(59 / 238)$ & $22.7 \%(27 / 119)$ & $26.9 \%(32 / 119)$ \\
$\begin{array}{c}\text { Waist } \\
\text { circumference }\end{array}$ & $10.1 \%(15 / 149)$ & $7.1 \%(5 / 70)$ & $12.7 \%(10 / 79)$ \\
$\begin{array}{c}\text { Systolic blood } \\
\text { pressure }\end{array}$ & $32.3 \%(50 / 155)$ & $33.9 \%(22 / 65)$ & $31.1 \%(28 / 90)$ \\
$\begin{array}{c}\text { Diastolic blood } \\
\text { pressure }\end{array}$ & $41.6 \%(64 / 154)$ & $36.9 \%(24 / 65)$ & $44.9 \%(40 / 89)$ \\
\hline
\end{tabular}

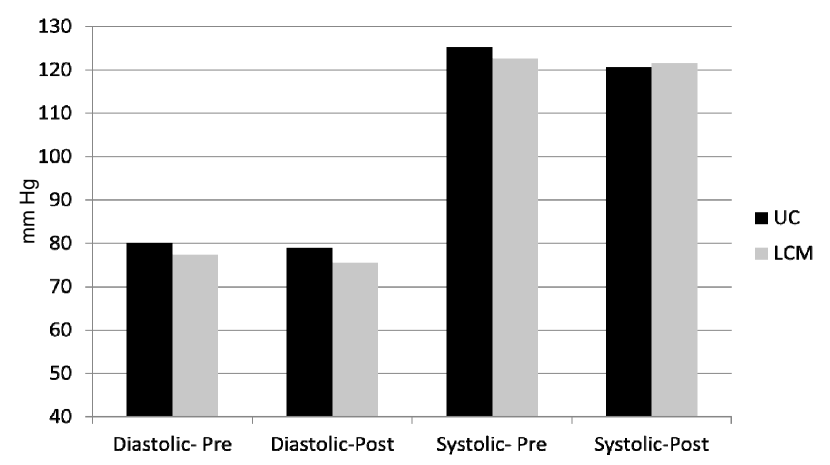

Figure 1. Mean blood pressure values pre and post.

vestment may have impeded quantity of data reporting.

\section{Conclusions}

This study shows that in an insured population RD counseling services in conjunction with a coordinated health promotion program result in improved health outcomes. Unfortunately, this study did not have an adequate sample size to show differences in health parameter outcome between the UC and LCM groups. Further study is needed to identify the specific aspects of MNT that contribute to changes in BMI, waist circumference and blood pressure.

\section{Acknowledgements}

Barbara Bapst, Denise Barratt, Susan Blomeley, Garen Brannon, Lisa Cooper, Kay Craven, Karen Donelson, Bonnie Elber, Karen Gantt, Sheila Garner, Susan Gregory, Janie Hoag, Barbara Ann Hughes, Johnnie Jackson, Carol Johnson, Kristen Ktecha, Jaime Lynn Lewis, Cathie Ostrowski, Karen Pfohl, Deborah Rosenquist, Jessica Seymour, Millicent Simmons, Rebecca Subbiah, Joye Willcox, Jan Foster, Dru Mueller, Donald Bradley.

\section{REFERENCES}

[1] L. Gaetke, M. Stuart and H. Truszczynska, “A Single Nutrition Counseling Session with a Registered Dietitian Improves Short-Term Clinical Outcomes for Rural Ken- 
tucky Patients with Chronic Diseases," Journal of the American Dietetic Association, Vol. 106, No. 1, 2006, pp. 109-112. doi:10.1016/j.jada.2005.09.051

[2] C. Lemon, K. Lacey, B. Lohse, D. Hubactier, B. Kiawitter and M. Palta, "Outcomes Monitoring of Health, Behavior, and Quality of Life after Nutrition Intervention in Adults with Type 2 Diabetes," Journal of the American Dietetic Association, Vol. 104, No. 12, 2004, pp. 18051815. doi:10.1016/j.jada.2004.09.024

[3] A. Holmes, B. Sanderson, R. Maisiak, A. Brown and V. Biitner, "Dietitian Services Are Associated with Improved Patient Outcomes and the MEDFICTS Dietary Assessment Questionnaire Is a Suitable Outcome Measure in Cardiac Rehabilitation," Journal of the American Dietetic Association, Vol. 105, No. 10, 2005, pp. 15331540. doi:10.1016/j.jada.2005.08.001

[4] K. Rhodes, L. Bookstein, L. Aaronson, N. Mercer and C. Orringer, "Intensive Nutrition Counseling Enhances Outcomes of National Cholesterol Education Program Dietary Therapy," Journal of the American Dietetic Association, Vol. 96, No. 10, 1996, pp. 1003-1010. doi:10.1016/S0002-8223(96)00268-4

[5] A. Wolf, M. Conaway, J. Crowther, K. Hazen, J. Nadler, B. Oneida and V. Bovbjerg, "Translating Lifestyle Intervention to Practice in Obese Patients with Type 2 Diabetes,” Diabetes Care, Vol. 27, No. 7, 2004, pp. 1570-1576. doi:10.2337/diacare.27.7.1570

[6] Centers for Disease Control and Prevention, "US Obesity Trends. Trends by State 1985-2008,” 2010.

http://www.cdc.gov/obesity/data/trends.html
[7] D. Howard, K. Thorpe and S. Busch, "Understanding Recent Increases in Chronic Disease Treatment Rates: More Disease or More Detection?" Health Economics, Policy and Law, Vol. 5, No. 4, 2010, pp. 411-435. doi:10.1017/S1744133110000149

[8] G. Oster, D. Thompson, J. Edelsberg, A. Bird and G. Colditz, "Lifetime Health and Economic Benefits of Weight Loss among Obese Persons,” American Journal of Public Health, Vol. 89, No. 10, 1999, pp. 1536-1542. doi:10.2105/AJPH.89.10.1536

[9] F. Pasanisi, F. Contaldo, G. de Simone and M. Mancini, "Benefits of Sustained Moderate Weight Loss in Obesity," Nutrition, Metabolism \& Cardiovascular Disease, Vol. 11, No. 6, 2001, pp. 401-406.

[10] Writing Group of the Nutrition Care Process/Standardized Language Committee, "Nutrition Care Process Part II: Using the International Dietetics and Nutrition Terminology to Document the Nutrition Care Process," Journal of the American Dietetic Association, Vol. 108, No. 8, 2008, pp. 1287-1293. doi:10.1016/j.jada.2008.06.368

[11] G. Murphy, L. Snetselaar, E. Myers, B. LaForge, L. Qualls, S. Blackwelder and D. Bradley, "Self-Reported Health Parameters Compared to Clinician Measurements: Methods in Practice Based Research,” Journal of Public Health Management and Practice, 2011 (unpublished).

[12] R. Miller and S. Rollnick, "Ten Things That Motivational Interviewing Is Not," Behavioural and Cognitive Psychotherapy, Vol. 37, No. 2, 2009, pp. 129-140. doi:10.1017/S1352465809005128 